\title{
Extended Heroin Access Increases Heroin Choices Over a Potent Nondrug Alternative
}

\author{
Magalie Lenoir ${ }^{1,2}$, Lauriane Cantin ${ }^{1,2,3}$, Nathalie Vanhille ${ }^{1,2,3}$, Fuschia Serre ${ }^{1,2}$ and Serge H Ahmed ${ }^{* 1,2}$ \\ 'Université de Bordeaux, Institut des Maladies Neurodégénératives, Rue Léo-Saignat, Bordeaux, France; ' ${ }^{2}$ CNRS, Institut des Maladies \\ Neurodégénératives, Rue Léo-Saignat, Bordeaux, France
}

\begin{abstract}
Epidemiological research shows that the proportion of drug users who become addicted to heroin is higher than to cocaine. Here we tested whether this difference could be due to a difference in the addiction liability between the two drugs. Addiction liability was assessed under a discrete-trials choice procedure by measuring the proportion of rats that prefer the drug over a potent alternative reward (ie, water sweetened with saccharin). Previous research on choice between self-administration of i.v. cocaine or sweet water showed that the proportion of cocaine-preferring rats remains relatively low and invariable (ie, I5\%), even after extended drug access and regardless of past drug consumption (ie, total drug use before choice testing). By contrast, the present study shows that under similar choice conditions, the proportion of heroin-preferring rats considerably increases with extended heroin access (6-9h per day for several weeks) and with past heroin consumption, from 11 to $51 \%$ at the highest past drug consumption level. At this level, the proportion of drug-preferring rats was about three times higher with heroin than with cocaine ( $51 \%$ vs $15 \%)$. This increase in the rate of heroin preference after extended heroin access persisted even after recovery from acute heroin withdrawal. Overall, these findings show that choice procedures are uniquely sensitive to different drugs and suggest that heroin is more addictive than cocaine. This higher addiction liability may contribute to explain why more drug users become addicted to heroin than to cocaine in epidemiological studies.

Neuropsychopharmacology (2013) 38, 1209-1220; doi:10.1038/npp.2013.17; published online 30 January 2013
\end{abstract}

Keywords: addiction liability; heroin; cocaine; choice; preference; alternative reinforcement.

\section{INTRODUCTION}

The transition to drug addiction only occurs in some drug users (Anthony, 2002). Epidemiological research shows that the proportion of drug users who develop addiction after drug experimentation can considerably vary with the drug used (Anthony et al, 1994; Anthony, 2002), suggesting that different drugs may possess different addiction liability. For instance, more drug users become addicted to heroin than to cocaine (Anthony et al, 1994; Anthony, 2002). However, as different drug users can choose different drugs, this difference in the rate of addiction between cocaine and heroin could reflect the characteristics of the drug users rather than, or in addition to, the differential addiction liability of these two drugs (Kliner and Pickens, 1982; Anthony, 2002; Kendler et al, 2012).

One classic way to address this problem has been to compare the reinforcing values of different drugs in

*Correspondence: Dr SH Ahmed Université de Bordeaux, Institut des Maladies Neurodégénératives, UMR 5293, I46 rue Léo-Saignat, Bordeaux F-33000, France. Tel: +33 557 57I 566, Fax: +33 556900 278, E-mail: sahmed@u-bordeaux2.fr

${ }^{3}$ These authors contributed equally to this work.

Received 20 November 2012; revised 24 December 20 I2; accepted 4 January 2013; accepted article preview online I5 January 2013 non-human animals under controlled laboratory conditions (Katz, 1990; Brady, 1991). These values can be measured using the progressive ratio schedule of reinforcement, which is considered as one of the best standard methods in the field (Arnold and Roberts, 1997). Using this method, cocaine (and amphetamine-like psychostimulants) is consistently found to maintain higher breaking points than heroin or morphine in laboratory rats, suggesting that cocaine would be more addictive than heroin (Richardson and Roberts, 1996; Ward et al, 2005). One well-known, though often overlooked, limitation of the progressive ratio procedure, however, is that it measures the net result of at least two different drug effects: the reinforcing value of the self-administered drug and its direct effects on performance. We recently found that the latter, value-independent effect contributes to the difference in breaking points between cocaine and heroin in rats. When this effect is minimized (ie, by imposing a minimum interval between successive drug self-injections), the breaking point of cocaine is considerably decreased (Cantin et al, 2010) while the breaking point of heroin is unaffected (Lauriane Cantin, Magalie Lenoir and Serge Ahmed, unpublished results). Thus, the progressive ratio procedure does not seem to be well-suited to compare the reinforcing values of cocaine and heroin. 
Alternative procedures that minimize the influence of drugs' value-independent effects during measurement of their reinforcing values are thus needed. Choice procedures involving a common, alternative nondrug reinforcer (eg, food) are particularly well-adapted for this purpose. These procedures have been extensively used in both human and non-human primates (Haney, 2009; Martinez et al, 2009; Walsh et al, 2010; Negus and Banks, 2011) but they are still largely understudied in rats (Ahmed, 2012; Kerstetter et al, 2012). In one recent series of discrete-trials choice studies, rats were allowed to choose between pressing a lever to get water sweetened with saccharin-a potent, albeit biologically inessential, nondrug reward-or an alternative lever to receive an intravenous dose of cocaine (Lenoir et al, 2007; Cantin et al, 2010; Augier et al, 2012). A potent rewarding alternative is necessary to insure that the opportunity cost of drug preference is sufficiently high. An equal level of effort was required on both levers and choice trials were sufficiently spaced to prevent the direct effects of cocaine at the moment of choice. Under these conditions, most rats chose almost exclusively the nondrug alternative (Lenoir et al, 2007; Cantin et al, 2010; Augier et al, 2012). This behavior was robust to a wide range of experimental conditions, including increasing drug doses per injection (Lenoir et al, 2007) and increasing levels of past drug consumption (ie, total drug consumption before choice testing; Cantin et al, 2010). At the highest level, only a minority of rats (about 15\%) preferred cocaine, even when the opportunity cost of not choosing the alternative was increased (Cantin $e t a l, 2010)$. As continued drug use at the expense of other rewarding activities and despite associated costs are hallmarks of drug addiction, we propose (i) that drug preference over a potent alternative reward may represent a valid measure of addiction in animals and (ii) that the proportion of drug-preferring rats may represent a good index of addiction liability: the higher this proportion for a given drug, the higher its addiction liability (Ahmed, 2010, 2012). In epidemiology, addiction liability is estimated by the proportion of drug users who become addicted after repeated drug use (Anthony et al, 1994; Anthony, 2002).

The overall goal of this study is to measure this index for heroin after extended heroin access and to compare it with the previously estimated index for cocaine. Since the discovery that heroin and cocaine self-administration partly depend on different neural substrates (Ettenberg et al, 1982; Koob, 1992), there has been cumulative evidence for addiction-related differences between these two drugs (Badiani et al, 2011; Koo et al, 2012), even after extended drug access (Lenoir et al, 2012). Notably, recent choice studies in monkeys demonstrated that while extended cocaine access had no effect on cocaine choices (Banks and Negus, 2010), extended heroin access considerably increased heroin choices, especially at the low doses (Negus, 2006; Negus and Rice, 2009). Overall, the present study extends to rats these findings. Contrary to extended cocaine access which had no significant impact on the proportion of cocaine-preferring rats (Lenoir et al, 2007; Cantin et al, 2010), extended heroin access considerably increased the proportion of heroin-preferring rats. This outcome is consistent with previous research showing that dependence and/or extended drug access can increase both heroin intake (Deneau et al, 1969; Ahmed et al, 2000; Chen et al, 2006; Vendruscolo et al, 2011) and motivation (Yanagita, 1973; Walker et al, 2003; Lenoir and Ahmed, 2008). At the highest level of past heroin consumption, the proportion of heroin-preferring rats was more than three times higher than that previously found with cocaine (ie, $51 \%$ vs $15 \%$ ) (Cantin et al, 2010). These convergent findings across species show that choice procedures are uniquely sensitive to different drugs and may suggest that heroin has a higher addiction liability than cocaine. The latter difference could contribute to the differential rate of heroin and cocaine addiction seen in human drug users (Anthony et al, 1994; Anthony, 2002).

\section{MATERIALS AND METHODS}

\section{Subjects}

Ninety seven adult male Wistar rats (Charles River, L'Arbresle, France, 249-340 g at the beginning of experiments) were used. Rats were housed in groups of 2-3 and maintained in temperature-controlled vivarium with a 12 -h light-dark cycle. Food and water were freely available in the home cages and rats were neither food- nor water-restricted during behavioral testing. All experiments were carried out in accordance with institutional and international standards of care and use of laboratory animals (UK Animals (Scientific Procedures) Act, 1986; and associated guidelines; the European Communities Council Directive (86/609/EEC, 24 November 1986) and the French Directives concerning the use of laboratory animals (décret 87-848, 19 October 1987)). A total of 80 rats completed this study and 17 rats did not for the following causes: death during intravenous surgery $(n=1)$; catheter failure $(n=5)$; infection $(n=4)$; failure to acquire or maintain operant behavior $(n=7)$.

\section{Surgery}

Anesthetized rats (Chloral hydrate, $500 \mathrm{mg} / \mathrm{kg}$ IP) (J-T Baker, The Netherlands) were surgically prepared with chronic silastic catheters (Dow Corning Corporation, Michigan, USA) in the right jugular vein. After surgery, catheters were flushed daily with $0.2 \mathrm{ml}$ of a sterile antibiotic solution containing heparinized saline $(280 \mathrm{IU} / \mathrm{ml})$ and ampicilline (Panpharma, Fougères, France). When a catheter leakage was suspected, the patency of the catheter was checked by an intravenous administration of etomidate (Etomidate Lipuro, $1 \mathrm{mg} / \mathrm{kg}$, Braun Medical, BoulogneBillancourt, France), a short-acting non-barbiturate anesthetic. Behavioral testing began 7-10 days after surgery.

\section{Apparatus}

Twelve identical operant chambers $(30 \times 40 \times 36 \mathrm{~cm})$ were used for all behavioral training and testing (Imetronic, Pessac, France). These chambers have been described in detail elsewhere (Augier et al, 2012). Briefly, each chamber was equipped with two automatically retractable levers (Imetronic), a commercially available lickometer circuit (Imetronic), two syringe pumps, a single-channel liquid swivel (Lomir Biomedical Inc, Quebec, Canada), and two pairs of infrared beams to measure horizontal cage crossings. 


\section{Standard Choice Procedure}

Rats were allowed to choose between a lever associated with heroin (lever $\mathrm{H}$ ) and a lever associated with water sweetened with $0.2 \%$ saccharin (lever $\mathrm{S}$ ) on a discrete-trials choice procedure similar to that used previously with cocaine (Lenoir et al, 2007; Cantin et al, 2010; Augier et al, 2012). Briefly, each daily choice session consisted of 10-12 discrete trials, spaced by 10-15 min, and divided into two successive phases, sampling (four trials) and choice (six or eight trials). During sampling, levers $\mathrm{S}$ and $\mathrm{H}$ were presented alternatively (in that order: $\mathrm{H}-\mathrm{S}-\mathrm{H}-\mathrm{S}$ ) to allow rats to separately evaluate each reward before making their choice. If rats responded within $5 \mathrm{~min}$ on the available lever, they were rewarded by the corresponding reward. Reward delivery was signaled by immediate retraction of the lever and brief illumination of a cue-light above it. If rats failed to complete the response requirement within $5 \mathrm{~min}$, the lever retracted and no cue-light or reward was delivered. During choice, both levers $\mathrm{S}$ and $\mathrm{H}$ were presented simultaneously allowing rats to choose mutually exclusively between the two to obtain the corresponding reward. Delivery of the chosen reward was signaled by simultaneous retraction of both levers and brief illumination of the cue-light above the selected lever. If rats failed to respond on either lever within $5 \mathrm{~min}$, both levers retracted and no cue-light or reward was delivered.

\section{Choice Between Heroin and Saccharin in Naive Rats}

A group of operant and drug naive rats $(n=12)$ was tested during 15 consecutive daily sessions under the choice procedure described above with 12 discrete trials, spaced by 10 min (four sampling trials followed by eight choice trials). The response requirement was initially set to 1 response (first 10 testing sessions) and then incremented to two consecutive responses to avoid eventual accidental choice (remaining sessions). Responding on lever S during sampling or choice was rewarded by a 20-s access to water sweetened with $0.2 \%$ of sodium saccharin delivered in an adjacent drinking cup. Responding on lever $\mathrm{H}$ was rewarded by one intravenous dose of $10 \mu \mathrm{g}$ (or about $30 \mu \mathrm{g} / \mathrm{kg}$ ) heroin in a volume of $0.074 \mathrm{ml}$ delivered over $2 \mathrm{~s}$.

\section{Choice Between Heroin and Saccharin in Operant- Trained Animals}

Before choice testing, a new group of rats $(n=18)$ was first trained for 4 weeks under a fixed-ratio 1 (FR1) schedule of saccharin and heroin self-administration on alternate daily sessions, 6 days a week as described previously (Cantin et al, 2010). Briefly, on saccharin sessions, only lever $S$ was available. Lever pressing on this lever was rewarded by a 20 -s access to water sweetened with $0.2 \%$ saccharin and initiated a 20-s time-out (TO) period signaled by the cue-light above lever S. On heroin sessions, only lever $\mathrm{H}$ was available. Lever pressing on this lever was rewarded by one intravenous dose of $10 \mu \mathrm{g}$ (or about $30 \mu \mathrm{g} / \mathrm{kg}$ ) heroin and initiated a 20 -s TO period signaled by the cue-light above lever $\mathrm{H}$. Sessions ended after rats had earned a maximum of 30 saccharin or heroin rewards or after $3 \mathrm{~h}$ had elapsed.
Effects of Drug dose on Choice Between Heroin and Saccharin

A subgroup of rats $(n=12)$ from the previous experiment was tested in a between-session manner with different unit doses of heroin (in that order: 10, 5, and $20 \mu \mathrm{g}$ delivered over $2 \mathrm{~s}$ ). To minimize differences between doses in heroin intoxication before each choice trial, the inter-trial interval was increased with the dose using the following method (Lenoir et al, 2007): typical inter-injection interval during continuous self-administration of the available dose (ie, 3.2, 5.3 , and $9.5 \mathrm{~min}$ for the dose of 5, 10, and $20 \mu \mathrm{g}$, respectively) plus $4.7 \mathrm{~min}$ (this constant added delay insured that rats that chose heroin on the previous trial were not drug-sated for the next trial). Each dose was tested for 4-5 consecutive sessions.

\section{Choice Between Heroin and Saccharin After Extended Heroin Access}

A separate group of naive rats were given daily 1-h (short access or ShA rats, $n=20$ ) or 6 -h access (long access or LgA $-6 \mathrm{~h}$ rats, $n=20)$ to heroin self-administration $(15 \mu \mathrm{g}$ per injection) as described previously (Lenoir and Ahmed, 2007, 2008). To speed up drug intake escalation in LgA-6 h rats, the unit dose of heroin during the last $5 \mathrm{~h}$ was increased fourfold to $60 \mu \mathrm{g}$ (or about $180 \mu \mathrm{g} / \mathrm{kg}$ ) by increasing the injection volume (Lenoir and Ahmed, 2007, 2008). Self-administration sessions were run 6 days per week.

After extended heroin access, all rats were first habituated to drink water sweetened with $0.2 \%$ saccharin for $1 \mathrm{~h}$ in the self-administration boxes (data not shown). During habituation, no levers were extended and rats could obtain saccharin by licking the cup ( $0.02 \mathrm{ml}$ per 10 licks). Then, rats were trained to press lever S under a FR1 TO $20 \mathrm{~s}$ schedule of saccharin reinforcement during six sessions. Saccharin sessions lasted $30 \mathrm{~min}$, except session 1 which lasted $1 \mathrm{~h}$, and alternated with regular 1 -h (ShA rats) or 6-h (LgA-6h rats) sessions of heroin self-administration. As a result, $\mathrm{LgA}-6 \mathrm{~h}$ rats had access to saccharin $18 \mathrm{~h}$ after withdrawal from extended heroin access.

After stabilization of responding for saccharin, all rats were allowed to choose between saccharin and heroin as described above except for the following minor procedural differences. Briefly, the choice phase consisted of six trials spaced by $15 \mathrm{~min}$ and the unit dose of heroin was $15 \mu \mathrm{g}$ (or about $45 \mu \mathrm{g} / \mathrm{kg}$ ). These minor differences, which are mainly due to historical reasons, had no significant impact on choice outcomes (see Results). Choice sessions alternated with regular 1-h (ShA rats) or 6-h (LgA-6 h rats) sessions of heroin self-administration. As a result, LgA-6h rats were allowed to choose between heroin and saccharin $18 \mathrm{~h}$ after withdrawal from extended heroin access (ie, $6+18=24 \mathrm{~h}$ ).

To assess the effects of an even more extended exposure to heroin on subsequent heroin choices, an additional group of rats $(n=10)$ was tested. These rats were allowed to self-administer heroin under a FR1 TO $20 \mathrm{~s}$ as described above for the LgA condition, except that the session length and the highest dose of heroin (ie, that available after the first hour) were further increased to $9 \mathrm{~h}$ and $120 \mu \mathrm{g}$ (or about $320 \mu \mathrm{g} / \mathrm{kg}$ ) per injection, respectively. After escalation of drug intake, rats were then trained to respond on lever $S$ 
for saccharin and then tested for choice as described in the above experiment. LgA-9 h rats had access to saccharin and choice sessions $15 \mathrm{~h}$ after withdrawal from extended heroin access (ie, $9+15=24 \mathrm{~h}$ ).

\section{Substitution of Heroin by Cocaine During Choice Between Drug and Saccharin}

A subgroup of LgA- 6 h rats $(n=5)$ tested in the first heroin intake escalation experiment was used in this preliminary drug substitution experiment. After choice testing between heroin and saccharin (see above), these rats were allowed to choose between i.v. cocaine $(250 \mu \mathrm{g}$ or about $750 \mu \mathrm{g} / \mathrm{kg})$ and saccharin for five additional sessions. Cocaine choice sessions consisted of eight choice trials spaced by $10 \mathrm{~min}$.

\section{Data Analysis}

All data were subjected to mixed analyses of variance, followed by post hoc comparisons using the Tukey's Honestly Significant Difference (HSD) test. The indifference level between saccharin and heroin was conveniently normalized at 0 in the choice procedure using the following formula: $1-(\%$ drug choices/50\%). Comparisons with the indifference level were conducted using a $t$-test. Proportions were compared using the two-proportion $z$-test. Finally, behavioral variables with ' 0 variance' were analyzed using non-parametric statistics (ie, Wilcoxon's test; Kruskal-Wallis).

\section{RESULTS}

When initially naive rats $(n=12)$ were allowed to choose between saccharin and heroin, they developed a preference for saccharin $(\mathrm{F}(14,154)=1.85, P<0.05)$ which stabilized after session 5 despite equal and near maximal sampling of both rewards over this time period $(\mathrm{F}(1,11)=3.66$, NS) (Figure 1a and $\mathrm{b}$ ). Interestingly and consistent with their preference, rats responded faster for saccharin than for heroin during sampling $(\mathrm{F}(1,11)=7.61, P<0.01$; Figure 1c). Similar findings were obtained in a separate group of rats $(n=18)$ that were trained on alternate sessions to selfadminister heroin and saccharin before choice testing.
After training, these rats tended to respond more for heroin than for saccharin $(\mathrm{F}(1,17)=1.27$, NS; Figure 2a) but they obtained slightly more saccharin rewards than heroin rewards $(\mathrm{Z}(18)=3.29, P<0.01$; Figure $2 \mathrm{~b})$. This apparent discrepancy was due to more TO responses for heroin than for saccharin $(6.9 \pm 0.9 v s 18.3 \pm 6.1)$. On average, rats took a total of $2.6 \pm 1.4 \mathrm{mg}$ (corresponding roughly to $7.7 \mathrm{mg} / \mathrm{kg}$ ) of heroin before choice testing. Such drug pre-exposure was not sufficient, however, to change rats' preference toward saccharin during choice testing (Figure 2c). Rats were indeed initially indifferent and then rapidly developed a robust preference for saccharin over heroin $(\mathrm{F}(7,119)=$ $8.68, P<0.01)$. Once saccharin preference was established, a subgroup of rats $(n=12)$ from this experiment continued to be tested with different doses of heroin. Though heroin induced a dose-dependent increase in locomotion $(\mathrm{F}(2,22)=9.62, P<0.01$; Figure $2 \mathrm{~d})$, preference score did not vary with the dose of heroin $(\mathrm{F}(2,22)=0.23$, NS; Figure 2e).

The previous choice experiments involved rats with no or limited exposure to heroin self-administration before choice testing. To assess the effects of more extended access to heroin for self-administration, a novel group of rats $(n=40)$ was given differential access to heroin for several weeks before choice testing. As expected from previous research, ShA rats maintained a low and stable level of heroin intake over time while LgA-6h rats kept escalating drug intake over the same period of time (Group $\times$ Session: $F(18,684)=$ 34.64, $P<0.01$; Supplementary Figure S1a). After escalation, LgA-6h rats took 13 times more heroin than ShA rats as averaged over the last three sessions $(1.47 \pm 0.07$ vs $0.11 \pm$ $0.01 \mathrm{mg}$ ). They also responded more than ShA rats for the same heroin dose, as measured during the first hour of the last three sessions $(11.2 \pm 0.7$ vs $22.1 \pm 1.6$ responses per hour; $\mathrm{F}(1,38)=29.35, P<0.01)$. In total, ShA rats took $2.0 \pm 0.1 \mathrm{mg}$ (or about $6.0 \mathrm{mg} / \mathrm{kg}$ ) while $\mathrm{LgA}-6 \mathrm{~h}$ took $21.9 \pm 1.0 \mathrm{mg}$ of heroin (or about $65.7 \mathrm{mg} / \mathrm{kg}$ ) over time. Extended access to heroin for self-administration was associated with a suppression of body mass growth compared with control access conditions (Group $\times$ Session: $\mathrm{F}(8,304)=$ 93.92, $P<0.01$; Supplementary Figure $S 1 b)$. When given repeated access to saccharin between heroin sessions,
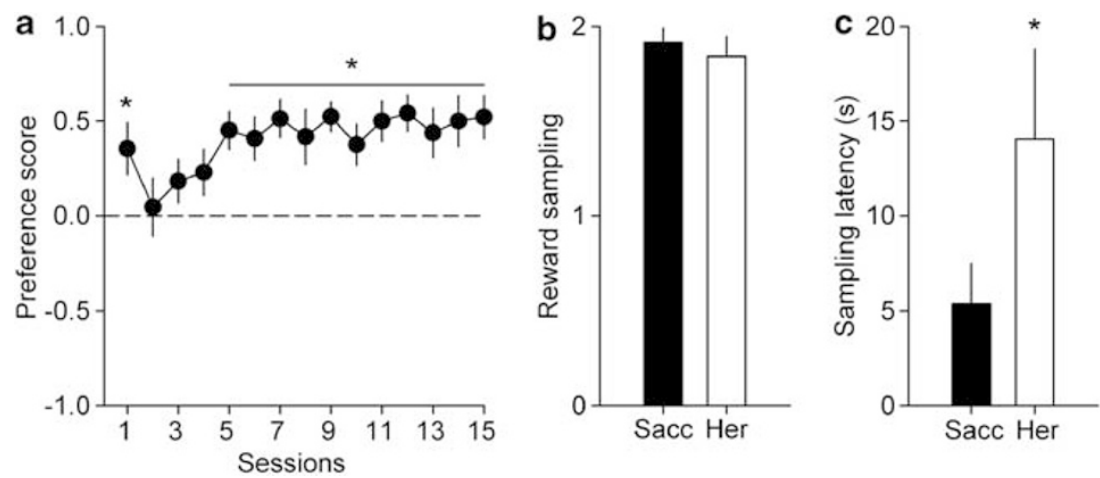

Figure I Choice between heroin and water sweetened with saccharin in naive rats $(n=12)$. (a) Mean preference scores ( \pm SEM) as a function of testing sessions. The horizontal dashed line at 0 represents the indifference level. Values above 0 indicate a preference for water sweetened with saccharin, while values below 0 indicate a preference for intravenous heroin. *Different from the indifference level $(P<0.05, t$-test). (b) Mean sampling ( \pm SEM) of lever $S$ (black bar) and lever $\mathrm{H}$ (white bar) (maximum possible per session $=2)$. (c) Bars represent mean ( \pm SEM) latencies of saccharin (black bar) and heroin (white bar) sampling (ie, time to complete the FR requirement after trial onset). *Different from saccharin $(P<0.05$, Tukey's HSD test). Data in (b) and (c) were obtained by averaging individual performances over the last three stable sessions. 
a

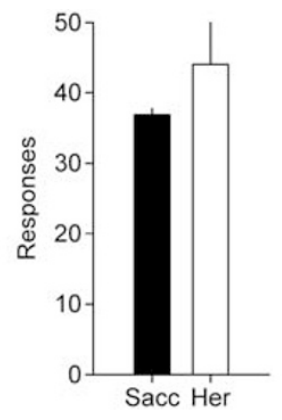

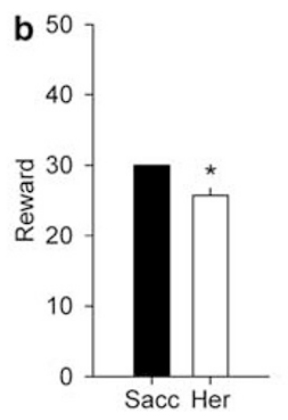
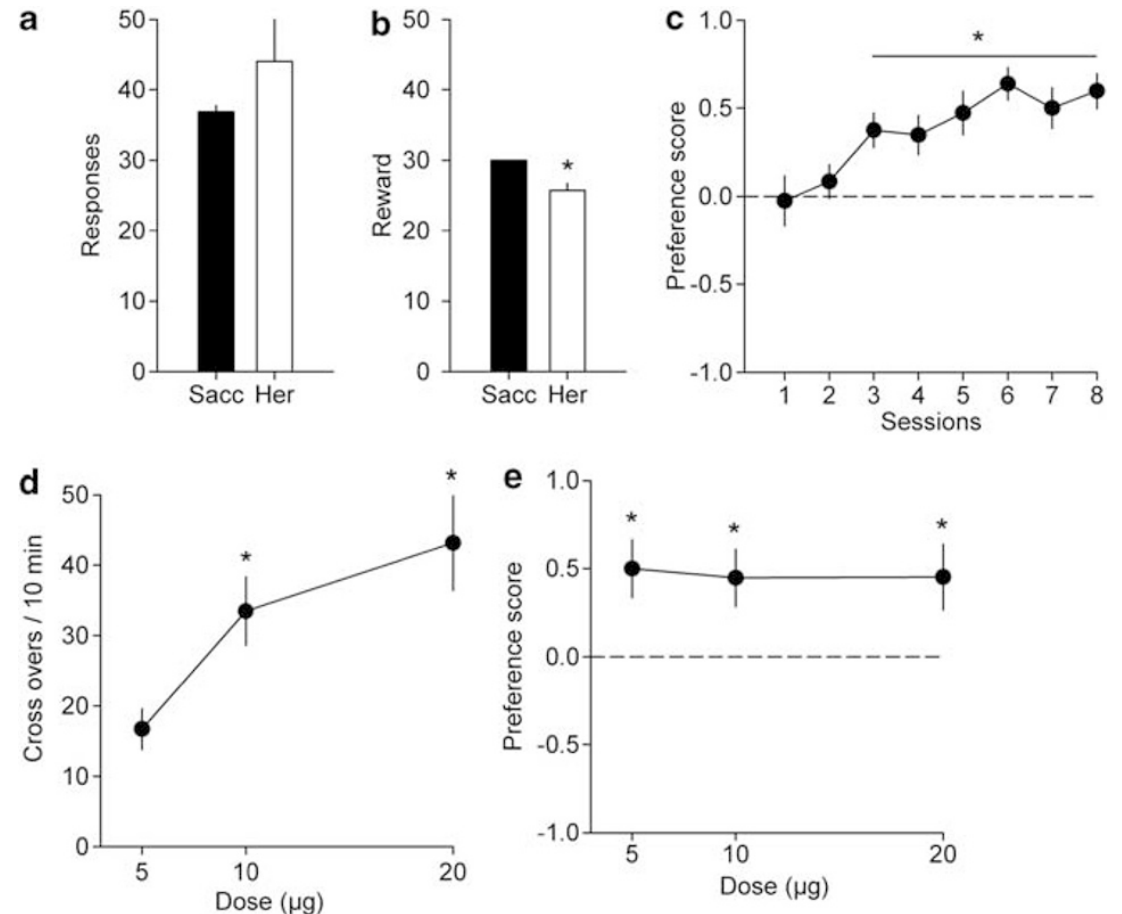

Figure 2 Choice between heroin and water sweetened with saccharin in operant-trained rats $(n=18)$. (a) Mean total responses ( \pm SEM) for saccharin (black bar) or heroin (white bar). (b) Mean numbers of earned heroin or saccharin rewards (maximum possible per session $=30$ ). *Different from saccharin $(P<0.05$, Wilcoxon's test). (c) Mean preference scores $( \pm$ SEM) as a function of testing sessions. For other information, see legend of Figure Ia. * Different from the indifference level $(P<0.05, t$-test). (d) Mean numbers $( \pm S E M)$ of cage crossings as a function of heroin doses. Cage crossings were measured during 10 min after the first heroin sampling. *Different from the lowest dose of heroin $(P<0.05$, Tukey's HSD test, $n=12)$. (e) Mean heroin choices as a function of heroin doses. *Different from the indifference level $(P<0.05, t$-test). All data, except in $(c)$, were obtained by averaging individual performances over the last three sessions.

LgA-6h rats responded less for saccharin (Group: $\mathrm{F}(1,38)=11.96, P<0.01$; Group $\times$ Session: $\mathrm{F}(5,190)=2.43$, NS; Figure 3a) and drank less of it than ShA rats (Group: $\mathrm{F}(1,38)=12.69, P<0.01$; Group $\times$ Session: $\mathrm{F}(5,190)=0.73$, NS; Figure $3 b$ ). When allowed to choose between the two rewards, ShA rats were initially indifferent but eventually developed a stable preference for saccharin while LgA- $6 \mathrm{~h}$ rats as a group remained indifferent $(\mathrm{F}(1,38)=8.20$, $P<0.01$ ) (Figure 3c). There was no significant difference between ShA and LgA-6h rats in completed choice trials $((\mathrm{F}(1,38)=2.98, \mathrm{NS})$, see Figure 4 below $)$ or in sampling behavior (Supplementary Figure S2). Importantly, though LgA- $6 \mathrm{~h}$ rats as a group were indifferent, $60 \%$ of them (ie, 12 out of 20) showed a preference for heroin (\% heroin choices $>50 \%$ of total choice trials over the last three sessions), a proportion of heroin-preferring individuals significantly higher than in the ShA group (60\% vs $25 \%, z=-2.2$, $P<0.05$, two-proportion $z$-test; Figure $3 \mathrm{~d}$ ).

To assess whether even more extended exposure to heroin could further increase heroin choices and heroin-preferring individuals, a separate group of rats $(n=10)$ was given 9-h access to a high dose of heroin (ie, $15 \mu \mathrm{g}$ per injection during the first hour followed by $120 \mu \mathrm{g}$ per injection during the last $8 \mathrm{~h}$ ). As expected, total heroin intake gradually escalated over time, from an initial mean level of $1.8 \pm$ $0.3 \mathrm{mg}$ per session to $4.8 \pm 0.9 \mathrm{mg}$ per session $(\mathrm{F}(20,180)=$ $11.30, P<0.01$; Supplementary Figure S3a). On average, LgA-9h rats took a total of $75.1 \pm 8.6 \mathrm{mg}$ of heroin (or about $225.3 \mathrm{mg} / \mathrm{kg}$ ) over time. This dramatic escalation of heroin self-administration was associated with an initial loss of body mass, followed by an almost complete suppression of body mass growth $(\mathrm{F}(20,180)=13.32, \quad P<0.01$; Supplementary Figure $\mathrm{S} 3 \mathrm{~b}$ ). When given repeated access to saccharin between heroin sessions, LgA-9 h rats progressively responded less for saccharin $(F(5,45)=10.70, P<0.01$; Figure 4a) and progressively drank less of it over time $(\mathrm{F}(5,45)=4.33, P<0.01$; data not shown). In the end, $\operatorname{LgA}-$ $9 \mathrm{~h}$ rats emitted fewer responses on lever $\mathrm{S}$ than both ShA and $\operatorname{LgA}-6 \mathrm{~h}$ rats from the previous experiment, as averaged over the last three sessions $(\mathrm{F}(2,47)=51.37, P<0.01$; inset in Figure 4a). When allowed to choose between heroin and saccharin, LgA- $9 \mathrm{~h}$ rats progressively completed less choice trials over time (Figure $4 \mathrm{~b}$ ) compared with both ShA and LgA-6 h rats (Group: $F(2,46)=19.41, P<0.01$; Group $\times$ Session: $F(4,92)=7.67, P<0.01)$. On the third choice session, the experiment had to be prematurely stopped because the percentage of completed trials per session (sampling + choice trials) dropped below $25 \%$, precluding a meaningful assessment of choice performance. As a result, LgA-9h took fewer doses of heroin than both ShA and LgA$6 \mathrm{~h}$ rats from the previous experiment as measured over the first three choice sessions $(F(2,47)=3.77, P<0.05$; inset in Figure $4 \mathrm{~b}$ ).

As rats were tested for choice $15 \mathrm{~h}$ after extended heroin access, this suppression of choice performance probably results from heroin withdrawal (HW). To test this hypothesis, extended heroin access was discontinued during 3 weeks during which rats were given daily access to 

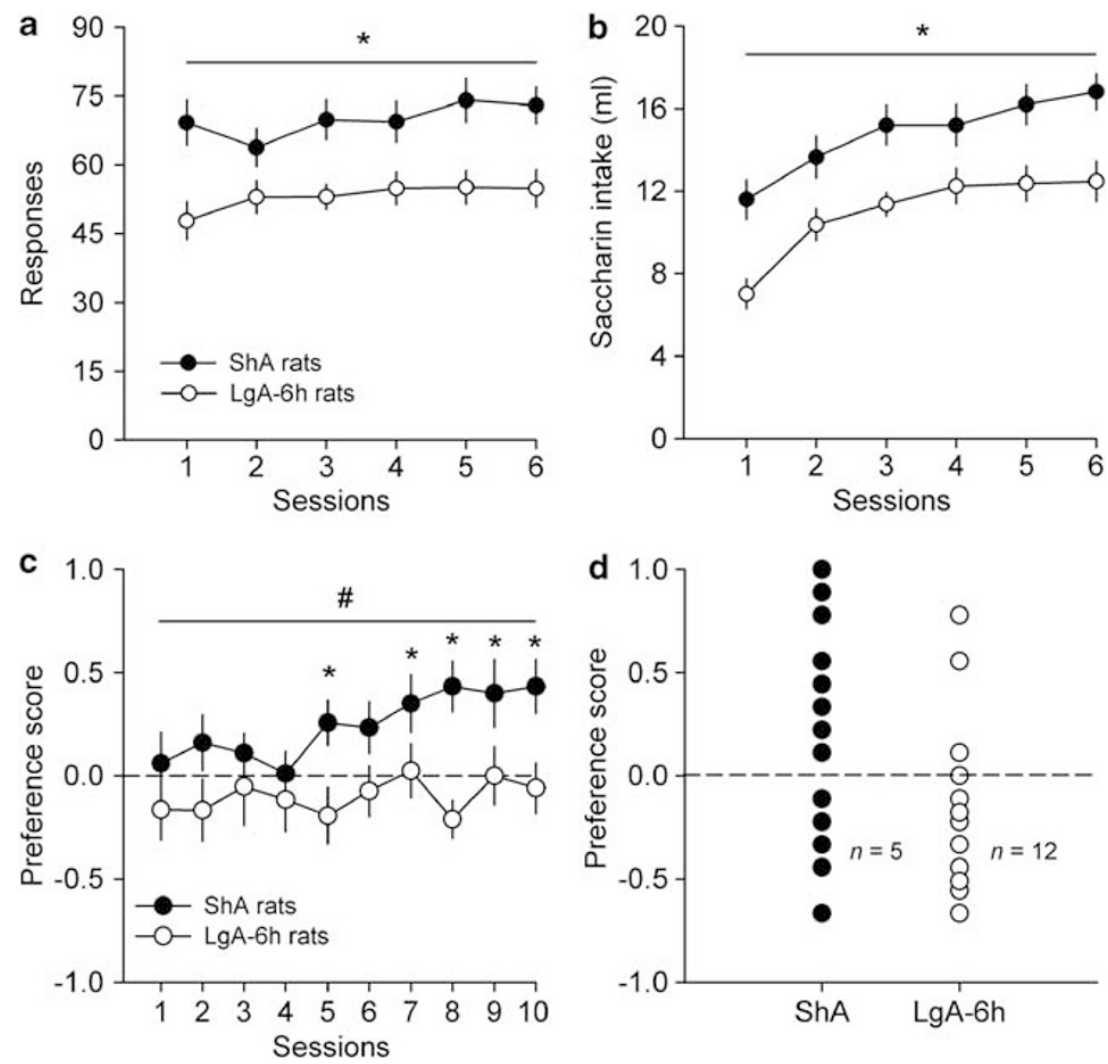

Figure 3 Choice between heroin and saccharin after extended heroin access. (a) Responding for and (b) intake of saccharin (ml) across sessions after differential access to heroin in ShA rats (closed circles, $n=20$ ) and LgA-6 h rats (open circles, $n=20)$. *Different from ShA rats ( $P<0.05$, Tukey's HSD test). Saccharin sessions alternated with sessions of heroin self-administration. (c) Mean ( \pm SEM) preference scores in both ShA and LgA-6h rats across repeated choice sessions. Choice sessions alternated with sessions of heroin self-administration. For other information, see legend of Figure Ia. *Different from the indifference level $\left(P<0.05\right.$, $t$-test). ${ }^{\#}$ Different from ShA rats $(P<0.05$, Tukey's HSD test). (d) Distribution of individual preferences in ShA and LgA-6h rats. Individual preferences were computed by averaging preference scores over the last three choice sessions. There were 5 and 12 heroin-preferring rats in the ShA and LgA groups, respectively $(P<0.05$, two-proportion z-test).

saccharin to allow recovery from HW. During the first 14 days, rats could obtain saccharin by licking the cup for $1 \mathrm{~h}$ per day (see procedure used for saccharin habituation); levers were retracted and no lever response was required to obtain saccharin. Then rats were retrained for five daily sessions to lever press under a FR1 TO $20 \mathrm{~s}$ schedule of saccharin reinforcement. As expected, rats drank progressively more saccharin during prolonged abstinence $(\mathrm{F}(13,117)=8.41, P<0.01$; Figure $4 \mathrm{c})$, reaching a volume of $15.3 \pm 2.5 \mathrm{ml}$ over the last three sessions. As a result, FR responding for saccharin, as averaged over the last three sessions, increased above responding measured during HW $(\mathrm{F}(2,18)=21.25, P<0.01$; Figure $4 \mathrm{~d})$ and recovered to the level of ShA rats (see Figure 3a). To directly assess the impact of acute HW on saccharin responding, rats were given one 9-h session of heroin self-administration and tested for saccharin responding $15 \mathrm{~h}$ later. As expected, acute withdrawal from extended heroin access (sHW) suppressed responding for saccharin (Figure $4 \mathrm{~d}$ ). Three days after recovery from $\mathrm{HW}, \mathrm{LgA}-9 \mathrm{~h}$ rats were retested for choice between heroin and saccharin during 10 consecutive sessions. They now completed virtually all trials across all choice sessions $(93.0 \pm 2.4 \%)$. As a group, LgA-9h rats were initially indifferent and remained so over repeated choice sessions $(F(9,81)=0.52$, NS; Figure 5a). At an individual level, however, $40 \%$ of them showed a preference for heroin as measured over the last three choice sessions (Figure 5b) - a proportion similar to that found in LgA$6 \mathrm{~h}$ rats tested during HW $(z=-1$, NS, two-proportion $z$ test). Finally, to assess the effects of HW on heroin choices, rats were given three 9-h sessions of heroin self-administration alternating with three choice sessions, with each choice session beginning $15 \mathrm{~h}$ after extended heroin access sessions. Performance during HW was compared with the last three preceding choice sessions. Heroin withdrawal non-selectively reduced the number of both saccharin and heroin sampling $(F(1,9)=11.86, P<0.01$, Figure $5 c)$ and the number of completed choice trials $(\mathrm{F}(1,9)=30.47, P<0.01$, Figure $5 \mathrm{~d}$ ) but had no effect on heroin choices $(\mathrm{F}(1,9)=$ 1.42 , NS, Figure 5e).

To further assess the impact of past heroin use on the frequency of heroin-preferring rats, a retrospective analysis of all choice experiments conducted in the laboratory, including unpublished studies, was performed. The total amount of self-administered heroin preceding choice testing was calculated for each individual $(n=191)$. This amount ranged from 0 to $169.8 \mathrm{mg}$ (or approximately $509.4 \mathrm{mg} / \mathrm{kg}$ ) and was positively correlated with heroin choices $(r=0.23, P<0.01)$. To further analyze the relationship between past drug consumption and drug choices, we 
a
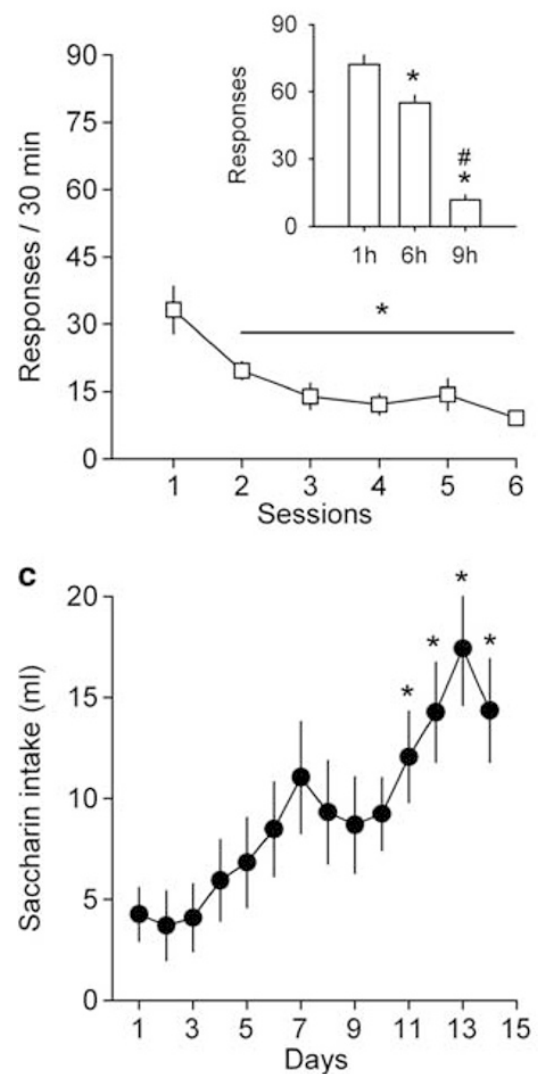

b $\quad-\square-\operatorname{LgA}-9 h$
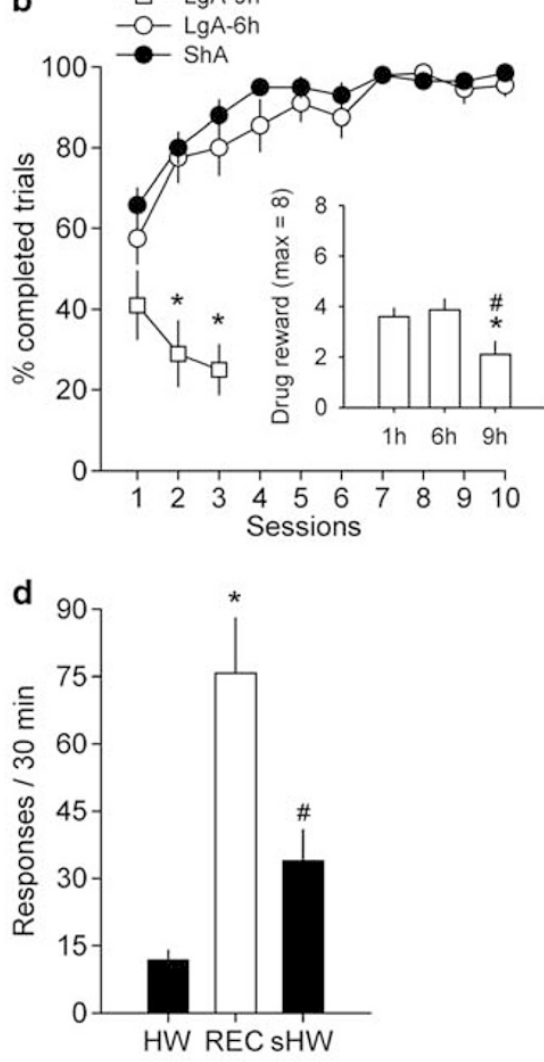

Figure 4 Effects of a more extended heroin access on saccharin responding and on choice between heroin and saccharin. (a) Mean ( \pm SEM) responses on lever $S$ in LgA-9 h rats $(n=10)$ during saccharin sessions. Saccharin sessions alternated with sessions of heroin self-administration. *Different from the first session ( $P<0.05$, Tukey's HSD test). (Inset in (a)) Mean responses ( \pm SEM of the last three sessions) on lever S by ShA, LgA-6h and LgA-9h rats. *Different from ShA rats $(P<0.05$, Tukey's HSD test). "Different from LgA-6h rats $(P<0.05$, Tukey's HSD test). (b) Percentage of completed trials ( \pm SEM) during the choice procedure between heroin and saccharin in ShA (closed circles), LgA-6h (open circles) and LgA-9h rats (open squares). *Different from ShA and LgA-6h rats $(P<0.05$, Tukey's HSD test). (Inset in (b)) Mean $( \pm S E M)$ number of earned heroin rewards. *Different from ShA rats $(P<0.05$, Tukey's HSD test). "Different from LgA-6h rats $(P<0.05$, Tukey's HSD test). (c) Saccharin intake in abstinent LgA-9h until recovery from HW. *Different from the first session ( $P<0.05$, Tukey's HSD test). (d) Mean responses ( \pm SEM) for saccharin during HW (last three sessions) and after recovery (REC, last three sessions). After recovery, rats were re-exposed to one single 9-h session of heroin self-administration and tested for saccharin responding $15 \mathrm{~h}$ later (s $\mathrm{HW}$ ). *Different from HW ( $P<0.05$, Tukey's HSD test). "Different from REC ( $P<0.0$ I, Tukey's HSD test).

defined four intervals or levels of past heroin consumption (ie, of $2.5 \mathrm{mg}$ each, except for the last open interval; Figure $6 \mathrm{a}$ and $\mathrm{b}$ ). With increased level of past heroin consumption, initial preference for saccharin gradually decreased and rats became indifferent between the two rewards $(F(3,18)=6.49, P<0.01$; Figure 6a). There was no systematic change in sampling behavior with increased past heroin use, except for a significant increase in saccharin sampling latency at the highest level of past drug use (Supplementary Figure S4). At the individual level, the proportion of heroin-preferring individuals significantly increased with past heroin consumption (Kruskal-Wallis, $\mathrm{H}(3,191)=16.32), P<0.01$; Figure $6 \mathrm{~b})$. At the highest level of past heroin consumption, $51 \%$ of rats preferred heroin over the alternative reinforcer. This proportion was significantly higher than the highest proportion of cocaine-preferring rats previously seen at the highest level of past cocaine consumption (Cantin et al, 2010) (51\% vs 15\%, $z=-3.2, P<0.01$, two-proportion $z$-test; Figure $6 \mathrm{~b})$. In fact, the proportion of heroin-preferring rats tended to be higher than the highest proportion of cocaine-preferring rats at all levels of past heroin consumption, except the lowest one.

Finally, to more directly compare heroin and cocaine under similar choice conditions, we performed a preliminary experiment in which heroin was substituted with cocaine in a small subgroup of $\operatorname{LgA}-6 \mathrm{~h}$ rats $(n=5)$. When heroin was available, rats as a group were indifferent (Figure 6c); however, after substitution with cocaine, the same rats rapidly developed an almost exclusive preference for saccharin (Drug: $\mathrm{F}(1,4)=19.19, \quad P<0.01$; $\quad$ Drug $\times$ Session: $F(4,16)=1.24, \mathrm{NS})$. Though only five rats were tested, all of them showed the same shift in drug choices, even those $(n=4)$ that initially preferred heroin (Figure $6 \mathrm{~d}$ ).

\section{DISCUSSION}

Contrary to extended cocaine access which had no effect on subsequent cocaine choices (Lenoir et al, 2007; Cantin et al, 2010), extended heroin access considerably increased the proportion of heroin-preferring rats from $11 \%$ at the lowest 

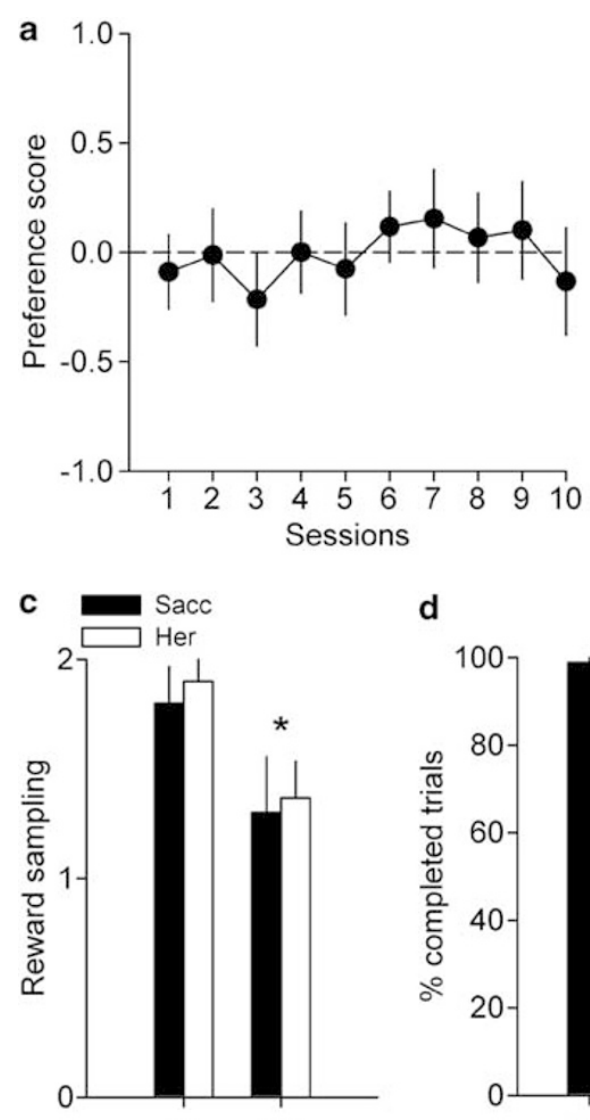

BL HW

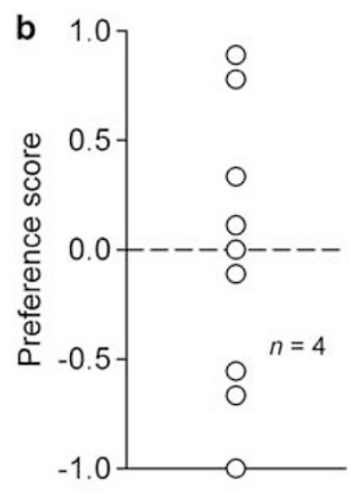

d

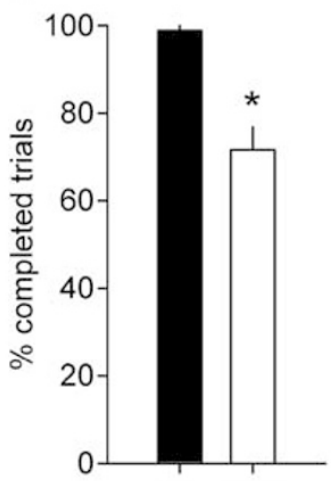

BL HW e

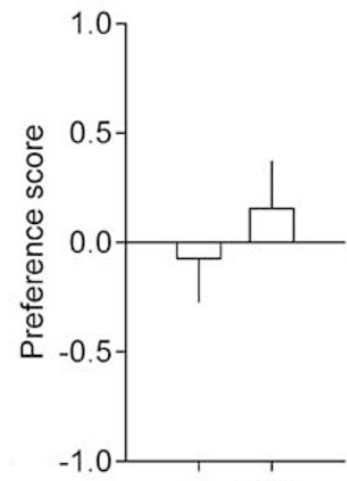

BL HW

Figure 5 Lack of effects of heroin withdrawal on choice between heroin and saccharin. (a) Mean ( \pm SEM) preference scores of $\operatorname{LgA}-9 \mathrm{~h}$ rats $(n=10)$ after recovery from HW. For other information, see legend of Figure la. (b) Distribution of individual preferences (averaged over the last three sessions, open circles) in LgA-9h rats. Four out of IO LgA-9h rats preferred heroin over saccharin. (c) Mean ( \pm SEM) saccharin (black bar) and heroin sampling (white bar) during post-recovery baseline $(\mathrm{BL})$ and after HW. (d) Mean $( \pm \mathrm{SEM})$ percentage of completed choice trials during post-recovery baseline $(\mathrm{BL})$ and after HW. (e) Mean ( \pm SEM) preference scores of rats during post-recovery baseline $(B L)$ and after HW. *Different from BL $(P<0.05$, Tukey's HSD test). Data in $(c-e)$ were obtained by averaging individual performances over the last three sessions of each condition.

level of past drug consumption (ie, initially naive animals) to $51 \%$ at the highest level. This study is consistent with previous research in monkeys showing that extended access to heroin, but not to cocaine self-administration, can dramatically increase subsequent drug choices (Negus, 2006; Negus and Rice, 2009; Banks and Negus, 2010). Overall, these convergent findings demonstrate that choice procedures are uniquely sensitive to drug differences and may suggest that heroin is more addictive than cocaine, though other interpretations are possible (see below). These findings add to growing evidence for significant addictionrelated differences between cocaine and heroin (Ettenberg et al, 1982; Koob, 1992; Badiani et al, 2011). Extending findings from monkeys to rats is important because this can open up new opportunities with significant impact on future addiction research. First, rats are generally more amenable than monkeys to invasive interventions for studying the neurobiology underlying drug choices and preferences, which is currently poorly understood. Second, rats can be tested in large cohorts, an advantage that can prove critical for studying some important features of addiction, such as, for instance, factors underlying individual vulnerability to addiction.

Extended heroin access and HW were associated with a robust escalation of heroin intake, as previously shown
(Deneau et al, 1969; Ahmed et al, 2000; Chen et al, 2006; Vendruscolo et al, 2011), and with the emergence of clear motivational markers of dependence (Chen et al, 2006), such as a blockade of normal body weight growth and a suppression of saccharin responding and intake. The latter phenomenon increased with past heroin consumption but gradually returned to normal after prolonged abstinence, confirming previous research in rats (Parker et al, 1973; Lieblich et al, 1991). In theory, HW could have contributed to increased heroin choices after extended heroin access by either increasing the reinforcing value of heroin or, alternatively, by decreasing the value of sweet water, or by producing both effects (Ahmed et al, 2000; Koob and Le Moal, 2001). Both conditioned and unconditioned heroin or morphine withdrawal has previously been demonstrated to increase responding for heroin in rats (Shaham et al, 1996; Kenny et al, 2006) and heroin choices in monkeys, especially at the lowest doses of heroin tested (Negus, 2006; Negus and Rice, 2009). However, there was no evidence for a role of $\mathrm{HW}$ in increased heroin choices in the present study as this increase persisted even after prolonged abstinence (ie, 3 weeks) and evidence for recovery of the reinforcing value of saccharin.

In fact, when HW was sufficiently intense (ie, in LgA-9h), it also suppressed choice performance (ie, number of 

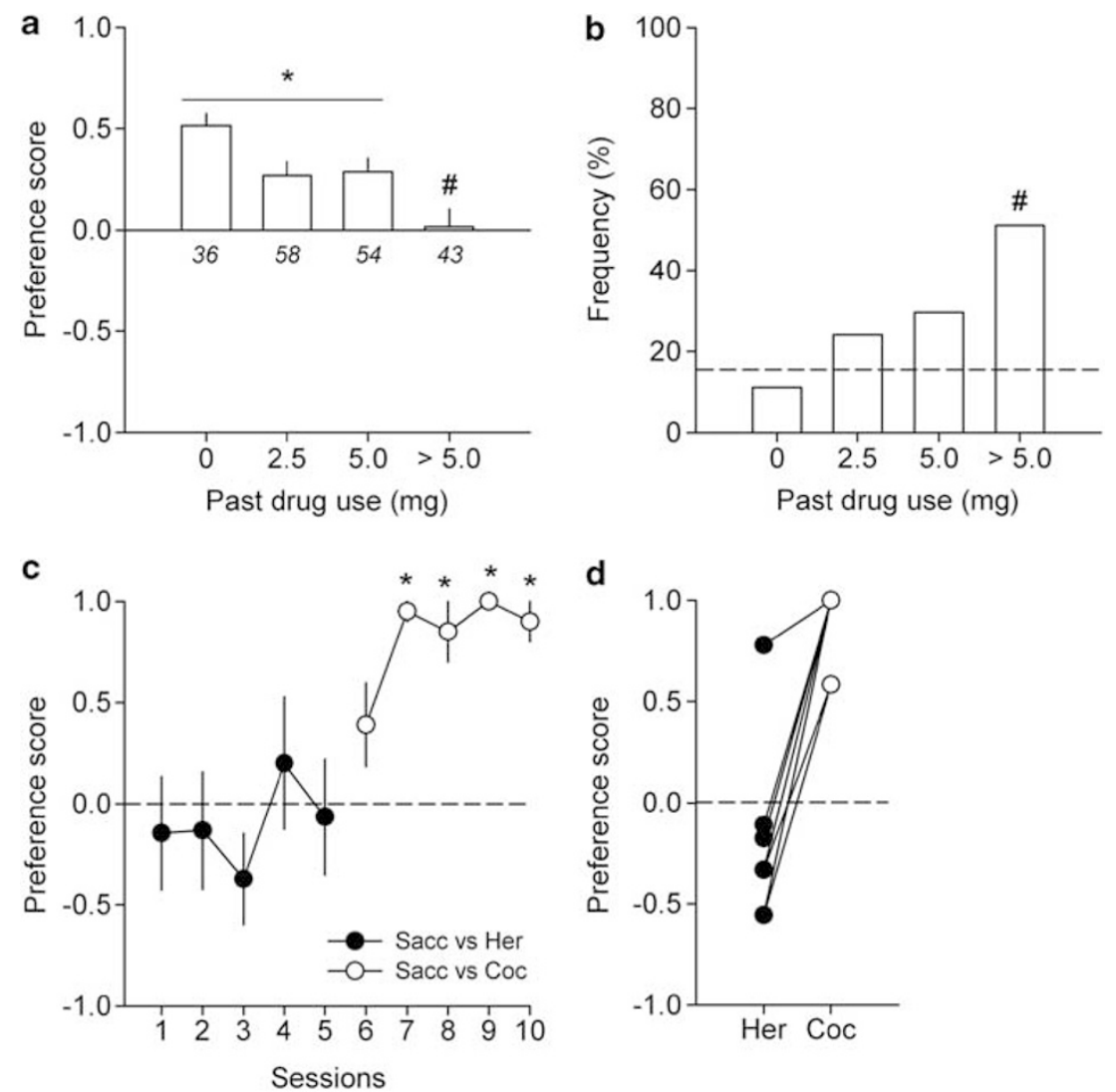

Figure 6 Effects of past heroin consumption on heroin choices. (a) Mean preference scores $( \pm$ SEM) over the last three sessions $(n=|9|)$ as a function of past heroin use. The number of rats per level of past heroin consumption is indicated below each bar. \#Different from the lowest level of past heroin consumption ( $P<0.05$, Tukey's HSD test). *Different from the indifference level $(P<0.05, t$-test). (b) Frequency of heroin-preferring individuals (ie, heroin choices $>50 \%$ of completed trials over the last three sessions) as a function of past heroin use. The horizontal dashed line at I5\% represents the frequency of cocaine-preferring individuals at the highest level of past cocaine use. "Different from the lowest level of past heroin consumption ( $P<0.05$, Tukey's HSD test). (c) Mean preference scores $( \pm$ SEM) of LgA-6h rats $(n=5)$ between heroin (closed circles) or cocaine (open circles) and saccharin. *Different from the indifference level $(P<0.05, t$-test). (d) Individual preferences (as averaged over the last three sessions) as a function of the available drug option.

completed trials), thereby paradoxically decreasing, not increasing, heroin responding and intake. These behaviorally debilitating effects, probably due to flu-like signs of acute withdrawal, are consistent with previous research using other behavioral procedures in rats (Hutcheson et al, 2001) and with recent evidence in heroin-withdrawn monkeys choosing between heroin and food (Negus, 2006; Negus and Rice, 2009). However, though monkeys completed less choice trials during HW, this effect was not associated with a decrease in heroin intake, as observed here in rats. In addition, decreased heroin intake during choice sessions is at odds with escalated levels of heroin intake seen in the same rats after the same period of HW (ie, 15-18 $\mathrm{h}$ after the preceding session of extended heroin access). One important factor that may explain this apparent discrepancy is that during choice sessions, heroin intake is limited every $10-15 \mathrm{~min}$, the first dose being available only after $10-15 \mathrm{~min}$. As a result, rats cannot take a sufficient amount of heroin to avoid the negative effects of HW on performance. By contrast, during sessions of extended heroin access, there is no temporal limitation on heroin intake, which allows rats to rapidly cumulate heroin intake to oppose or prevent the negative effects of HW (Kenny et al, 2006). As HW intensifies with repeated exposure to extended heroin access, this self-regulation process is supposed to drive heroin intake escalation (Ahmed et al, 2000; Koob and Le Moal, 2001; Kenny et al, 2006). Thus, discrete-trials choice procedures may not be well suited to study acute HW in rats because of their sensitivity to its behaviorally debilitating effects. However, these procedures may be particularly amenable to study protracted affective withdrawal, which can go on for weeks (in rats) and months to years (in humans). Protracted affective withdrawal may explain why heroin preference persists after dissipation of the debilitating effects of acute withdrawal in the present study. At a neurobiological level, there is evidence that protracted affective withdrawal is associated with increased activity in brain stress systems, such as corticotrophin-releasing factor and norepinephrine systems. Chronic activation of these brain systems has been hypothesized to increase the reinforcing value of opiates via negative reinforcement and to enhance the vulnerability to relapse after prolonged abstinence (Koob, 2008). Importantly, however, chronic activation of brain stress pathways does not always negatively interfere with food reward (Dallman, 2012), which may explain why, in the present study, increased heroin choices persisted during protracted withdrawal despite recovery of saccharin intake.

When compared with similar research on cocaine (Lenoir et al, 2007; Cantin et al, 2010; Augier et al, 2012), the 
present study shows that after extended drug access, more rats prefer heroin to saccharin (ie, 51\%) than cocaine to the same nondrug reward (ie, 15\%). If individual drug preference despite the opportunity of making a different choice reflects an addiction-like state, then this large difference may suggest that heroin is more addictive than cocaine (Anthony et al, 1994; Anthony, 2002). This conclusion is reinforced by the outcome of our pilot drug substitution study showing that when cocaine is substituted to heroin, heroin-preferring rats rapidly and almost exclusively switch their choice to sweet water. This conclusion is also consistent with other research that have compared the reinforcing value of cocaine and heroin using procedures that minimize the direct effects of drugs on performance. For instance, rats' responding for heroin before the first drug injection is generally higher than rats' responding for cocaine (Arroyo et al, 1998; Alderson et al, 2000; Everitt and Robbins, 2000; Lenoir et al, 2012). Rats also run faster and without hesitation in a runway to get heroin than to get cocaine (Ettenberg and Geist, 1993). The hypothesis that heroin is more addictive than cocaine is contradicted, however, by other research showing that rats prefer cocaine over heroin when facing a choice between the two drugs (Ward et al, 2005; Caprioli et al, 2009). In one study, virtually all rats (15 out of 16) preferred exclusively cocaine to heroin, regardless of the doses available for choice (Ward et al, 2005). The origin of this discrepancy between studies is currently unknown and deserves additional investigation.

Perhaps the difference between heroin and cocaine in the rate of drug preference is due to the specific kind of nondrug options available (ie, water sweetened with saccharin) rather than, or in addition to, the addiction liability of the drug per se. There are more synergies and commonalities between sweet reward and opiate drugs, including heroin, than between sweet reward and cocaine (Berridge, 2003; Avena et al, 2008; Kenny, 2011). First, sweet water palatability and/or consumption are enhanced by heroin or morphine, but they are unchanged or suppressed by cocaine (Woolverton et al, 1978; Cooper, 1982; Parker et al, 1992; Pecina and Berridge, 1995). Second, there are also evidence for cross-tolerance and cross-dependence between opiates and sweet food or drink after chronic exposure (Lieblich et al, 1983; Colantuoni et al, 2002). Third, intake of sweet water before withdrawal onset can prevent some of the physical symptoms of opiate withdrawal in morphine-dependent rats (Jain et al, 2004). Finally, at the neurobiological level, both sweet and heroin rewards depend on nucleus accumbens opioid signaling and are only weakly influenced by midbrain dopamine neurons (Ettenberg et al, 1982; Koob, 1992; Pecina and Berridge, 1995; Zhang and Kelley, 1997). The nucleus accumbens contains 'sweet hotspots' that amplify responses to sweetness in rats (Pecina et al, 2006; Smith and Berridge, 2007; Smith et al, 2011). Opioid modulation of these hotspots amplifies both palatability of and motivation for sweet reward while dopamine modulation of the same hotspots only amplifies motivation for sweet reward. If heroin induces an opioid-like and cocaine a dopamine-like modulation of accumbal hotspots, as one would expect given their respective pharmacology, then this may partly explain the difference between these two drugs in our choice procedure. However, it is not clear how such differential drug modulation of sweet hotspots should translate into more heroin choices than cocaine choices rather than the opposite. Nevertheless, because sweet reward has more similarities and synergies with heroin than with cocaine, it is possible that a different pattern of cocaine and heroin choices would be obtained with a different nondrug reward (eg, exercise, sex, or non-aggressive social interaction). Finally, contrary to heroin, cocaine can have, in addition to its rewarding effects, anxiogenic effects, especially in initially drug-naive rats (Ettenberg and Geist, 1991). Avoidance of these effects may contribute to the difference in drug choices between heroin and cocaine and may explain the rapid increase in sweet choices when heroin was substituted by cocaine in the present study. However, this explanation is unlikely because diazepam-a benzodiazepine that blocks the initial anxiogenic effects of cocaine (Ettenberg and Geist, 1991)-did not increase cocaine choices (Augier et al, 2012), as one would expect if rats refrained from choosing cocaine because of its anxiogenic effects.

Several potential limitations to this study need to be discussed. First, as explained above, rats cannot control the rate of drug consumption during discrete-trials choice testing and thus cannot attain or maintain their preferred level of intake. The inability to attain or maintain drug intake at a preferred level may expose rats to the delayed opponent affective effects of drugs that can follow their immediate rewarding effects (Ettenberg, 2004), thereby possibly making drug choices more ambivalent than saccharin choices. This factor is unlikely, however, to have a significant role in our procedure, at least in the case of cocaine, because blockade of the delayed negative effects of cocaine by diazepam (Ettenberg, 2004) did not increase drug choices (Augier et al, 2012). Second, our choice procedure is apparently not sensitive to drug doses (present study; Lenoir et al, 2007), contrary to other choice procedures in monkeys (Negus, 2006). Clearly, this lack of sensitivity is not due to the dose range tested, because the same dose range can produce significant dose-dependent effects on other behavioral outcomes, including locomotion (present study; (Ahmed and Cador, 2006; Lenoir and Ahmed, 2007; Lenoir et al, 2007), reinstatement of drug seeking after extinction (Ahmed and Cador, 2006; Lenoir and Ahmed, 2007), and rate of drug self-administration (Lenoir et al, 2012). The apparent lack of dose sensitivity of our choice procedure is more likely due to the large difference in reward value between the drug and saccharin, a difference that was intended to be difficult to surmount to increase the validity of drug preference as a measure of addiction in animals (see Introduction). We previously showed that rats can shift their preference toward cocaine only when the value of saccharin is considerably decreased, either by a 30-fold decrease in saccharin concentration or by a 10-fold increase in saccharin cost (Cantin et al, 2010). Choice procedures in monkeys were perhaps more sensitive to drug doses because the difference in reward value between drugs (cocaine, heroin) and food was less pronounced than in the present study. Finally, as discussed above, acute withdrawal from the more extended access to heroin (ie, $9 \mathrm{~h}$ per session) decreased heroin choices, an effect opposite to what one would expect if rats were seeking to minimize the negative impact of withdrawal and to what has been found in monkeys (Negus, 2006). This different outcome may 
suggest that rats are more sensitive than rhesus monkeys to the behaviorally debilitating effects of acute withdrawal and/or that rats are less prone than monkeys to learn to associate heroin use with the relief of HW.

Overall, the present study shows that the proportion of heroin-preferring rats increases with extended heroin access and is much higher than the highest proportion of cocainepreferring rats as measured under similar choice conditions. We interpret this difference as evidence that heroin has a higher addiction liability than cocaine, a difference that may, at least partly, contribute to the differential rate of heroin and cocaine addiction observed in human drug users (Anthony et al, 1994; Anthony, 2002). However, more research is needed to test the generality of this hypothesis across a wide range of individual and environmental factors susceptible to influence drug preferences (Caprioli et al, 2009; Kerstetter et al, 2012).

\section{ACKNOWLEDGEMENTS}

We thank Stephane Lelgouach for animal care, Pierre Gonzalez for technical assistance, Marie-Hélène Bruyères and Ourida Gaucher for administrative assistance, Christian Darrack for his help with data extraction, and Alain Labarriere for house-keeping assistance. This work was supported by Centre National de la Recherche Scientifique (CNRS), Agence Nationale de la Recherche (ANR), Mission Interministérielle de Lutte contre la Drogue et la Toxicomanie (MILDT), Fondation pour la Recherche Médicale (FRM), Université Bordeaux-Segalen, and Conseil Regional d'Aquitaine. We thank Dr Karine Guillem and Dr Sylvia Navailles, and Youna Vandaele for their comments on the manuscript. Finally, we also thank the reviewers for their criticisms.

\section{DISCLOSURE}

The authors declare no conflict of interest.

\section{REFERENCES}

Ahmed SH (2010). Validation crisis in animal models of drug addiction: beyond non-disordered drug use toward drug addiction. Neurosci Biobehav Rev 35: 172-184.

Ahmed SH (2012). The science of making drug-addicted animals. Neuroscience 211: 107-125.

Ahmed SH, Cador M (2006). Dissociation of psychomotor sensitization from compulsive cocaine consumption. Neuropsychopharmacology 31: 563-571.

Ahmed SH, Walker JR, Koob GF (2000). Persistent increase in the motivation to take heroin in rats with a history of drug escalation. Neuropsychopharmacology 22: 413-421.

Alderson HL, Robbins TW, Everitt BJ (2000). Heroin selfadministration under a second-order schedule of reinforcement: acquisition and maintenance of heroin-seeking behaviour in rats. Psychopharmacology (Berl) 153: 120-133.

Anthony JC (2002). Epidemiology of drug dependence. In: Davis KL, Charney D, Coyle JT, Nemeroff C (eds) Neuropsychopharmacology: the Fifth Generation of Progress. Lippincott Williams and Wilkins: Philadelphia, pp 1557-1573.

Anthony JC, Warner LA, Kessler RC (1994). Comparative epidemiology of dependence on tobacco, alcohol, controlled substances, and inhalants: basic findings from the National Comorbidity Survey. Exp Clin Psychopharmacol 2: 224-268.

Arnold JM, Roberts DC (1997). A critique of fixed and progressive ratio schedules used to examine the neural substrates of drug reinforcement. Pharmacol Biochem Behav 57: 441-447.

Arroyo M, Markou A, Robbins TW, Everitt BJ (1998). Acquisition, maintenance and reinstatement of intravenous cocaine selfadministration under a second-order schedule of reinforcement in rats: effects of conditioned cues and continuous access to cocaine. Psychopharmacology (Berl) 140: 331-344.

Augier E, Vouillac C, Ahmed SH (2012). Diazepam promotes choice of abstinence in cocaine self-administering rats. Addict Biol 17: 378-391.

Avena NM, Rada P, Hoebel BG (2008). Evidence for sugar addiction: behavioral and neurochemical effects of intermittent, excessive sugar intake. Neurosci Biobehav Rev 32: 20-39.

Badiani A, Belin D, Epstein D, Calu D, Shaham Y (2011). Opiate versus psychostimulant addiction: the differences do matter. Nat Rev Neurosci 12: 685-700.

Banks ML, Negus SS (2010). Effects of extended cocaine access and cocaine withdrawal on choice between cocaine and food in rhesus monkeys. Neuropsychopharmacology 35: 493-504.

Berridge KC (2003). Pleasures of the brain. Brain Cogn 52: 106-128.

Brady JV (1991). Animal models for assessing drugs of abuse. Neurosci Biobehav Rev 15: 35-43.

Cantin L, Lenoir M, Augier E, Vanhille N, Dubreucq S, Serre F et al (2010). Cocaine is low on the value ladder of rats: possible evidence for resilience to addiction. PLoS One 5: e11592.

Caprioli D, Celentano M, Dubla A, Lucantonio F, Nencini P, Badiani A (2009). Ambience and drug choice: cocaine- and heroin-taking as a function of environmental context in humans and rats. Biol Psychiatry 65: 893-899.

Chen SA, O'Dell LE, Hoefer ME, Greenwell TN, Zorrilla EP, Koob GF (2006). Unlimited access to heroin self-administration: independent motivational markers of opiate dependence. Neuropsychopharmacology 31: 2692-2707.

Colantuoni C, Rada P, McCarthy J, Patten C, Avena NM, Chadeayne A et al (2002). Evidence that intermittent, excessive sugar intake causes endogenous opioid dependence. Obes Res 10: 478-488.

Cooper SJ (1982). Palatability-induced drinking after administration of morphine, naltrexone and diazepam in the non-deprived rat. Subst Alcohol Actions Misuse 3: 259-266.

Dallman MF (2012). Stress-induced obesity and the emotional nervous system. Trends Endocrinol Metab 21: 159-165.

Deneau G, Yanagita T, Seevers MH (1969). Self-administration of psychoactive substances by the monkey. Psychopharmacologia 16: $30-48$.

Ettenberg A (2004). Opponent process properties of self-administered cocaine. Neurosci Biobehav Rev 27: 721-728.

Ettenberg A, Geist TD (1991). Animal model for investigating the anxiogenic effects of self-administered cocaine. Psychopharmacology (Berl) 103: 455-461.

Ettenberg A, Geist TD (1993). Qualitative and quantitative differences in the operant runway behavior of rats working for cocaine and heroin reinforcement. Pharmacol Biochem Behav 44: 191-198.

Ettenberg A, Pettit HO, Bloom FE, Koob GF (1982). Heroin and cocaine intravenous self-administration in rats: mediation by separate neural systems. Psychopharmacology (Berl) 78: 204-209.

Everitt BJ, Robbins TW (2000). Second-order schedules of drug reinforcement in rats and monkeys: measurement of reinforcing efficacy and drug-seeking behaviour. Psychopharmacology (Berl) 153: 17-30.

Haney M (2009). Self-administration of cocaine, cannabis and heroin in the human laboratory: benefits and pitfalls. Addict Biol 14: 9-21. 
Hutcheson DM, Everitt BJ, Robbins TW, Dickinson A (2001). The role of withdrawal in heroin addiction: enhances reward or promotes avoidance? Nat Neurosci 4: 943-947.

Jain R, Mukherjee K, Singh R (2004). Influence of sweet tasting solutions on opioid withdrawal. Brain Res Bull 64: 319-322.

Katz JL (1990). Models of relative reinforcing efficacy of drugs and their predictive utility. Behav Pharmacol 1: 283-301.

Kendler KS, Chen X, Dick D, Maes H, Gillespie N, Neale MC et al (2012). Recent advances in the genetic epidemiology and molecular genetics of substance use disorders. Nat Neurosci 15: 181-189.

Kenny PJ (2011). Common cellular and molecular mechanisms in obesity and drug addiction. Nat Rev Neurosci 12: 638-651.

Kenny PJ, Chen SA, Kitamura O, Markou A, Koob GF (2006). Conditioned withdrawal drives heroin consumption and decreases reward sensitivity. J Neurosci 26: 5894-5900.

Kerstetter KA, Ballis MA, Duffin-Lutgen S, Carr AE, Behrens AM, Kippin TE (2012). Sex differences in selecting between food and cocaine reinforcement are mediated by estrogen. Neuropsychopharmacology 37: 2605-2614.

Kliner DJ, Pickens R (1982). Indicated preference for drugs of abuse. Int J Addict 17: 543-547.

Koo JW, Mazei-Robison MS, Chaudhury D, Juarez B, LaPlant Q, Ferguson D et al (2012). BDNF is a negative modulator of morphine action. Science 338: 124-128.

Koob GF (1992). Drugs of abuse: anatomy, pharmacology and function of reward pathways. Trends Pharmacol Sci 13: 177-184.

Koob GF (2008). A role for brain stress systems in addiction. Neuron 59: 11-34.

Koob GF, Le Moal M (2001). Drug addiction, dysregulation of reward, and allostasis. Neuropsychopharmacology 24: 97-129.

Lenoir M, Ahmed SH (2007). Heroin-induced reinstatement is specific to compulsive heroin use and dissociable from heroin reward and sensitization. Neuropsychopharmacology 32: 616-624.

Lenoir M, Ahmed SH (2008). Supply of a nondrug substitute reduces escalated heroin consumption. Neuropsychopharmacology 33: 2272-2282.

Lenoir M, Serre F, Cantin L, Ahmed SH (2007). Intense sweetness surpasses cocaine reward. PLoS One 2: e698.

Lenoir M, Guillem K, Koob GF, Ahmed SH (2012). Drug specificity in extended access cocaine and heroin self-administration. Addict Biol 17: 964-976.

Lieblich I, Yirmiya R, Liebeskind JC (1991). Intake of and preference for sweet solutions are attenuated in morphinewithdrawn rats. Behav Neurosci 105: 965-970.

Lieblich I, Cohen E, Ganchrow JR, Blass EM, Bergmann F (1983). Morphine tolerance in genetically selected rats induced by chronically elevated saccharin intake. Science 221: 871-873.

Martinez D, Slifstein M, Narendran R, Foltin RW, Broft A, Hwang $\mathrm{DR}$ et al (2009). Dopamine D1 receptors in cocaine dependence measured with PET and the choice to self-administer cocaine. Neuropsychopharmacology 34: 1774-1782.

Negus SS (2006). Choice between heroin and food in nondependent and heroin-dependent rhesus monkeys: effects of naloxone, buprenorphine, and methadone. J Pharmacol Exp Ther 317: 711-723.
Negus SS, Rice KC (2009). Mechanisms of withdrawal-associated increases in heroin self-administration: pharmacologic modulation of heroin vs food choice in heroin-dependent rhesus monkeys. Neuropsychopharmacology 34: 899-911.

Negus SS, Banks ML (2011). Making the right choice: lessons from drug discrimination for research on drug reinforcement and drug self-administration. In: Glennon RLYoung $\mathrm{R}$ (eds)Drug Discrimination: Applications to Medicinal Chemistry and Drug StudiesJohn Wiley and Sons, Inc.: New York, pp 361-388.

Parker L, Failor A, Weidman K (1973). Conditioned preferences in the rat with an unnatural need state: morphine withdrawal. J Comp Physiol Psychol 82: 294-300.

Parker LA, Maier S, Rennie M, Crebolder J (1992). Morphine- and naltrexone-induced modification of palatability: analysis by the taste reactivity test. Behav Neurosci 106: 999-1010.

Pecina S, Berridge KC (1995). Central enhancement of taste pleasure by intraventricular morphine. Neurobiology (Bp) 3: 269-280.

Pecina S, Smith KS, Berridge KC (2006). Hedonic hot spots in the brain. Neuroscientist 12: 500-511.

Richardson NR, Roberts DC (1996). Progressive ratio schedules in drug self-administration studies in rats: a method to evaluate reinforcing efficacy. J Neurosci Methods 66: 1-11.

Shaham Y, Rajabi H, Stewart J (1996). Relapse to heroin-seeking in rats under opioid maintenance: the effects of stress, heroin priming, and withdrawal. J Neurosci 16: 1957-1963.

Smith KS, Berridge KC (2007). Opioid limbic circuit for reward: interaction between hedonic hotspots of nucleus accumbens and ventral pallidum. J Neurosci 27: 1594-1605.

Smith KS, Berridge KC, Aldridge JW (2011). Disentangling pleasure from incentive salience and learning signals in brain reward circuitry. Proc Natl Acad Sci USA 108: E255-E264.

Vendruscolo LF, Schlosburg JE, Misra KK, Chen SA, Greenwell TN, Koob GF (2011). Escalation patterns of varying periods of heroin access. Pharmacol Biochem Behav 98: 570-574.

Walker JR, Chen SA, Moffitt H, Inturrisi CE, Koob GF (2003). Chronic opioid exposure produces increased heroin self-administration in rats. Pharmacol Biochem Behav 75: 349-354.

Walsh SL, Donny EC, Nuzzo PA, Umbricht A, Bigelow GE (2010). Cocaine abuse versus cocaine dependence: cocaine self-administration and pharmacodynamic response in the human laboratory. Drug Alcohol Depend 106: 28-37.

Ward SJ, Morgan D, Roberts DC (2005). Comparison of the reinforcing effects of cocaine and cocaine/heroin combinations under progressive ratio and choice schedules in rats. Neuropsychopharmacology 30: 286-295.

Woolverton WL, Kandel D, Schuster CR (1978). Tolerance and cross-tolerance to cocaine and d-amphetamine. J Pharmacol Exp Ther 205: 525-535.

Yanagita T (1973). An experimental framework for evaluation of dependence liability of various types of drugs in monkeys. Bull Narc 25: 57-64.

Zhang M, Kelley AE (1997). Opiate agonists microinjected into the nucleus accumbens enhance sucrose drinking in rats. Psychopharmacology (Berl) 132: 350-360.

Supplementary Information accompanies the paper on the Neuropsychopharmacology website (http://www.nature.com/npp) 